\title{
Factores físicos que influyen en las relaciones florísticas de los piñonares (Pinaceae) de San Luis Potosí, México
}

\author{
Angélica Romero, Mario Luna \& Edmundo García \\ Postgrado en Botánica, Colegio de Postgraduados, Campus Montecillo. Carretera México-Texcoco, km 36.5, \\ Montecillo, Texcoco, Estado de México.56230. México: dahly@colpos.mx,mluna@colpos.mx, \\ edmundo@colpos.mx
}

\author{
Recibido 22-VII-2013. C Corregido 08-XI-2013. Aceptado 04-XII-2013.
}

\begin{abstract}
Physical factors influencing the floristic relationships of pinyon pine (Pinaceae) from San Luis Potosi, Mexico. In plant communities, the species distribution patterns and their relationships with environmental factors are of central importance in ecology. In San Luis Potosi of Mexico, woodlands of Pinus cembroides and $P$. johannis are sympatric, but $P$. cembroides tends to be located in South and Southwest slopes, in more disturbed sites; unlike, $P$. johannis, is mostly distributed in mesic areas, in North and Northeast slopes. The aim of this study was to analyze the influence of some physical factors on the floristic similarity of pinyon pine $P$. cembroides and P. johannis. The study area was located in the Sierra San Miguelito, San Luis Potosi, Mexico. We selected 40 sampling units spread over an area of $50 \mathrm{~km}^{2}$. In each unit, we laid out two $20 \mathrm{~m}$ long lines perpendicular to each other, in which we recorded cover data of the plant species intercepted. We developed two data matrices, the first one including cover values of 91 species, and the second one, considering seven topographical, climatic, and solar radiation variables. We applied cluster analysis and ordination to explore the influence of environmental variables on the floristic differentiation of pinyon pine woodlands. Clustering showed six groups, the first three characterized by $P$. cembroides. The ordination showed that variance represented by the first three axes was $65.9 \%$. Axis 1 was positively correlated with altitude and negatively with mean annual temperature; axes two and three, showed low correlation with the variables tested. P. cembroides woodlands and accompanying flora tend to be located in lower altitude, higher mean annual temperature, and mainly in South-Southwestern slopes. In contrast, stands of $P$. johannis, mixed stands of $P$. johannis-P. cembroides, and Quercus potosina, were usually founded in greater altitudes, mean annual temperature slightly lower, and NorthNortheastern exposure. The sites of these monospecific and mixed woodlands with associated species, indicators of environmental variables, generates the pre-diagnosis of the situation of the communities and their condition, so that future actions can be planned in agreement with the natural balance of the ecosystem. Rev. Biol. Trop. 62 (2): 795-808. Epub 2014 June 01.
\end{abstract}

Key words: pinyon pine, flora, cluster, ordination, indicator species.

Los patrones de distribución-abundancia de las especies en el paisaje y el conocimiento de los factores que controlan su distribución, han sido temas de interés para los ecólogos. Las formaciones vegetales responden a gradientes de factores como el clima, topografía y suelo (ter Braak \& Prentice, 1988; Légaré, Bergeron, Leduc \& Paré, 2001). A escala local, la pendiente y orientación de los sitios influye notablemente en la composición de las comunidades vegetales, debido a interacciones con el ángulo de la pendiente sobre la luz disponible, la incidencia calórica y los niveles de humedad del suelo (Parker, 1991; Korb, Daniels, Laughlin \& Fule, 2007).

Los piñonares (Pinus, Subgénero Strobus, sección Parrya, subsección Cembroides) están conformados por las comunidades dominadas principalmente por Pinus cembroides Zucc., y, en algunas localidades, por $P$. johannis (M.F. Robert), dentro del Altiplano Mexicano, al sur del Desierto Chihuahuense. Los piñonares 
son importantes en términos fitogeográficos y ecológicos, ya que México es el segundo centro de diversidad de piñoneros (Lanner, 1981), favorecido por la heterogeneidad y el nivel de aislamiento del paisaje, inclusive, varias especies de piñoneros y táxones acompañantes son endémicos de México (Romero-Manzanares, García-Moya \& Passini, 1996). Adicionalmente, éstas comunidades forestales ofrecen leña, madera, forraje, albergue para la fauna nativa y, en cuestión de servicios ambientales, influyen en los procesos hidrológicos, edáficos y atmosféricos, al mitigar gases invernadero (Braasch, 2012), hechos que realzan la importancia de su prevalencia. Social y económicamente, los piñonares han sido fuente de abasto de piñones desde tiempos ancestrales; actualmente, hay demanda de estas semillas para venta en el mercado nacional y extranjero por el uso que tienen los piñones en confitería, especialmente los de la especie $P$. cembroides.

Con respecto a los bosques identificados como piñonares, Aldrete \& Aguirre (1982), Passini (1982, 1994), Hernández-Reyna \& García-Moya (1985), Romero-Manzanares, García-Moya, Luna-Cavazos \& De la RosaVázquez (1989) realizaron inventarios en comunidades forestales simpátricas dominadas por $P$. cembroides y P. johannis, de ellos, hay quienes mencionan sobre la amplia semejanza florística entre comunidades. En contraste, hemos observado que aun cuando los piñonares sean simpátricos, $P$. cembroides tiende a distribuirse en las exposiciones sur-suroeste de las laderas y en sitios más deteriorados, mientras que los bosques de $P$. johannis se localizan en áreas mésicas, sobre las laderas con exposición norte-noreste y en sitios con regular a buena condición. Suponemos que la transición xéricomésica persistente entre el Altiplano Semiárido y las Serranías Meridionales en San Luis Potosí, México, provoca variaciones microclimáticas indirectamente causadas por cambios en topografía, altitud, exposición y substrato, las cuales a su vez, favorecen el establecimiento de especies indicadoras asociadas a los piñonares puros y mixtos de P. cembroides, P. johannis y sus combinaciones con Quercus potosina Trel.
Para analizar las relaciones entre diferentes sitios con desigual composición florística y estructura de las comunidades, se ha utilizado el análisis multivariable; el resultado orienta hacia el reconocimiento de los factores del medio que influyen en la estructura de la vegetación $\mathrm{y}$, permite conocer los patrones de distribución de las especies (Sardinero, 2000; Moora et al., 2007), examinar elementos y áreas florísticas (McLaughlin, 1994), agrupar táxones de distribución similar para correlacionarlos con variables del medio (Birks, 1987) y priorizar áreas para la conservación (Justus \& Sarkar, 2002). El objetivo de este estudio fue analizar la semejanza en la composición florística de los piñonares de $P$. cembroides y $P$. johannis, mediante la clasificación y ordenación de la vegetación acompañante, apoyada con herramientas multivariables. La hipótesis es que a $P$. cembroides le acompañan especies de hábito xérico propias del medio seco, mientras que a $P$. johannis se le asocian especies características de hábito mésico, menos tolerantes a la sequía. Consideramos que con esta investigación contribuiríamos a la comprensión de las relaciones florísticas que establecen diferencias en bosques fitogeográficamente simpátricos y, a la vez, aportaríamos nuevos indicios sobre los factores del medio, que al presente, condicionan la composición florística de los piñonares mexicanos.

\section{MATERIALES Y MÉTODOS}

Área de estudio: El área de estudio se ubica en la Sierra San Miguelito-Carranco, Provincia Biogeográfica del Altiplano Sur Zacatecano-Potosino (CONABIO, 1997); entre los 22॰00'2"94" - 22॰04'8" $12^{\prime} " \mathrm{~N}$ y $101^{\circ} 04^{\prime} 10^{\prime \prime} 03$ " - 10109'01'05" W, cercana al poblado La Amapola, ejido Escalerillas, San Luis Potosí, S.L.P. La altitud varía entre 1950-2 800m y la pendiente oscila de 5-50\%. El sustrato es riolítico y el relieve es de serranías con dirección noroeste-sureste y pisos de valle (Labarthe, Tristán, \& Aranda, 1982); los cerros son angulosos, escarpados y fracturados, mientras que los llanos tienen depósitos de aluvión. 
El suelo que predomina es Litosol, menos de $25 \mathrm{~cm}$ de profundidad, de textura media FAOUNESCO-ISRIC (1998). El clima es semiseco templado con lluvias en verano, transicional entre los menos secos de los secos $\left(\mathrm{BS}_{1}\right)$ y templados subhúmedos (CW) (INEGI, 2002); la evaporación sobrepasa a la precipitación. En cuanto a la vegetación, predominan bosques de $P$. cembroides y $P$. johannis, en masas puras, mixtas o asociadas con $Q$. potosina.

La recolección del piñón es una de las actividades de aprovisionamiento más importantes de la región, aunque también se aprovechan otras especies de flora y fauna silvestre. El uso de los árboles también es para construcción, combustible y sombra para la producción animal que se sustenta de arbustivas y herbáceas (Romero-Manzanares, Aguirre-Rivera, \& García- Moya, 1999). Entre los problemas de La Amapola S.L.P. y el área forestal que le rodea, destacan la tendencia a la desertización, erosión hídrica severa, reducción de la infiltración y recarga hidrológica, ciclos biogeoquímicos disminuidos, cambios en el balance energéticohidrológico y ramoneo del ganado, que en conjunto reducen la densidad de la vegetación en crecimiento (Huber-Sannwald et al., 2012).

Datos de campo y análisis de la información: La información de campo fue tomada durante el verano de 2011 en 40 sitios de muestreo seleccionados al azar, distribuidos en una superficie de aproximadamente $50 \mathrm{~km}^{2}$. Trabajamos con el método de línea Canfield y para ello, en cada uno de los 40 sitios se trazaron dos líneas interceptrices de $20 \mathrm{~m}$, perpendiculares entre sí, produciendo en total 80 líneas; por intervalos de $2 \mathrm{~m}$ para todas las líneas, se registraron tanto la especie que era tocada por la línea, como las medidas del intercepto (dosel sobrepuesto a la línea) y la amplitud (dosel perpendicular al intercepto). Adicionalmente, se efectuó la recolecta de material botánico para la determinación de las especies, el cual se depositó en el Herbario-Hortorio del Colegio de Postgraduados-CHAPA.

Con el propósito de evaluar semejanzas y diferencias, así como la influencia de los factores ambientales medidos en la composición florística de los piñonares, se elaboraron dos matrices de datos; una primera matriz usada para el análisis de grupos, consistió de las 40 unidades de muestreo y los valores de cobertura de 91 especies registradas; para los análisis numéricos se utilizaron sólo 70 táxones, el resto se eliminaron pues sólo aparecieron en menos del 5\% de las unidades de muestreo y se consideran como especies raras, de acuerdo con el criterio de selección de especies de McCune \& Grace (2002). Una segunda matriz consistió de los 40 sitios de muestreo y siete variables topográficas, climáticas y de incidencia solar, como son: altitud (m s.n.m), azimut (ubicación del sitio de muestreo en grados con referencia al norte, en el sentido de las manecillas del reloj), pendiente (grados), temperatura media anual $\left({ }^{\circ} \mathrm{C}\right)$, evapotranspiración $(\mathrm{mm})$, índice de radiación solar estimado con base en el ángulo de la pendiente, la latitud (en grados decimales) del sitio muestreado y la orientación de la pendiente e índice de calor. La primera y segunda matrices fueron utilizadas para un análisis de ordenación, con el propósito de explorar la influencia de las variables ambientales en el patrón de diferenciación florística de los piñonares del área de estudio.

Clasificación y ordenación de las comunidades: Se realizó un análisis de agrupamiento entre las 40 unidades de muestreo con base en la matriz de cobertura de las especies; el propósito fue analizar la semejanza en composición florística, y corroborar la relación entre las unidades de muestreo, derivada de la ordenación. Se eligió el Índice de Sørensen como el coeficiente de distancia entre grupos, por robusto y porque es una de las medidas de similitud más efectivas entre muestras o especies (McCune \& Grace, 2002); para el ligamiento de grupos se seleccionó el procedimiento Beta Flexible, porque conserva la distancia entre las unidades de muestreo (Peck, 2010). Los resultados se muestran en un dendrograma donde las medidas de distancia cambian como una función objetiva (Wishart, 1969), que disminuye el número de conjuntos formados e 
incrementa un máximo de información retenida, de manera que los grupos se construyen con base en la homogeneidad de las unidades de muestreo. También se aplicó el procedimiento de permutación de respuesta múltiple (MRPP, por sus siglas en inglés) con el propósito de evaluar las diferencias florísticas de los grupos derivados del análisis de agrupamiento, bajo la hipótesis nula de que no existen diferencias florísticas entre esos grupos. Detalles del procedimiento MRPP pueden ser consultados en Mielke (1991) y McCune \& Grace (2002); en este análisis, el estadístico $\mathrm{T}$ describe la diferencia entre los grupos bajo la premisa de que a medida que $\mathrm{T}$ es más negativa, entonces la diferencia entre grupos será mayor (Lesica, McCune, Cooper \& Hong, 1991).

La ordenación se llevó a cabo mediante escalamiento multidimensional no-métrico (EMNM), procedimiento efectuado para explicar la estructura de la comunidad y analizar los patrones de diferenciación de las unidades de muestreo. El EMNM se efectuó de acuerdo con el método descrito por Kruskal (1964) y el procedimiento mencionado por Peck (2010) y McCune \& Mefford (2011); aplicamos la medida de distancia de Sørensen, por ser robusta para estimar distancia ecológica (Beals, 1984). El EMNM es un método efectivo de ordenación, adecuado a datos de distribución no-normal o discontinuos de comunidades ecológicas (McCune \& Grace, 2002); además que tiene la propiedad de preservar el orden de disimilitud entre muestras de acuerdo con el rango de distancias entre ellas (Clarke, 1993). El procedimiento general inició con el cálculo del número de dimensiones a ser consideradas para explicar la estructura de los datos y una prueba de aleatorización para la solución requerida, así como la determinación del valor de estrés, el cual estima la confiabilidad al interpretar los resultados de la ordenación. Una vez seleccionado el número de dimensiones para la solución final, tres en el presente caso, se procedió a un nuevo análisis con 250 iteraciones, de las cuales se eligió como solución final, a aquélla con el menor estrés (Peck, 2010). La relación entre las especies y las variables ambientales se evaluó mediante correlaciones de estos atributos con los ejes de ordenación. Los análisis descritos se efectuaron con el programa PCORD 6 (McCune \& Mefford, 2011).

\section{RESULTADOS}

\section{Composición florística e importancia} de las especies del piñonar: Se registraron 91 especies en el área. El 29.1\% de las especies lo representaron plantas menores a $25 \mathrm{~cm}$ mientras que el $60.2 \%$ estuvo conformado por individuos de entre 25 y $100 \mathrm{~cm}$, y el restante $10.7 \%$ lo integraron elementos de tamaño mayor a $1 \mathrm{~m}$ de altura. Las familias mejor representadas pertenecieron a los estratos intermedios del bosque, ellas fueron Asteraceae, Poaceae, Lamiaceae, Fabaceae, Euphorbiaceae y Cyperaceae. En el bosque monoespecífico de $P$. cembroides, Poaceae fue muy importante, mientras que en el bosque de P. johannis, además de las Poaceae hubo representantes de Asteraceae, Lamiaceae y Fabaceae. Lo mismo se observó en el bosque mixto, pero cuando la asociación incluyó $Q$. potosina, predominaron las Poaceae y Asteraceae. En el apéndice quedó listado el elenco florístico.

Clasificación: Mediante el análisis de clasificación se reconocieron seis grupos a un nivel de $25 \%$ de información remanente (Fig. 1). Los tres primeros grupos estuvieron conformados por piñonares de $P$. cembroides, salvo dos casos que representaron un sitio de $P$. cembroides-P. johannis y otro de $P$. johannis- $Q$. potosina; en ellos, las especies más representativas fueron Tagetes micrantha, Microchloa kuntii, Lycurus phleoides, Bouteloua gracilis, Dichondra argentea, Jatropha dioica, Allium glandulosum, Dalea bicolor y Aristida divaricata. El cuarto grupo comprendió un piñonar puro de $P$. cembroides y dos asociaciones de este con $P$. johannis y $Q$. potosina más una de $P$. johanis- $Q$. potosina; en estos sobresalen especies como T. micrantha, Muhlenbergia pubescens, Aristida schiedeana, Echeandia flavescens, Cyperus seslerioides, Crusea diversifolia, Desmodium grahamii y Piptochaetium 


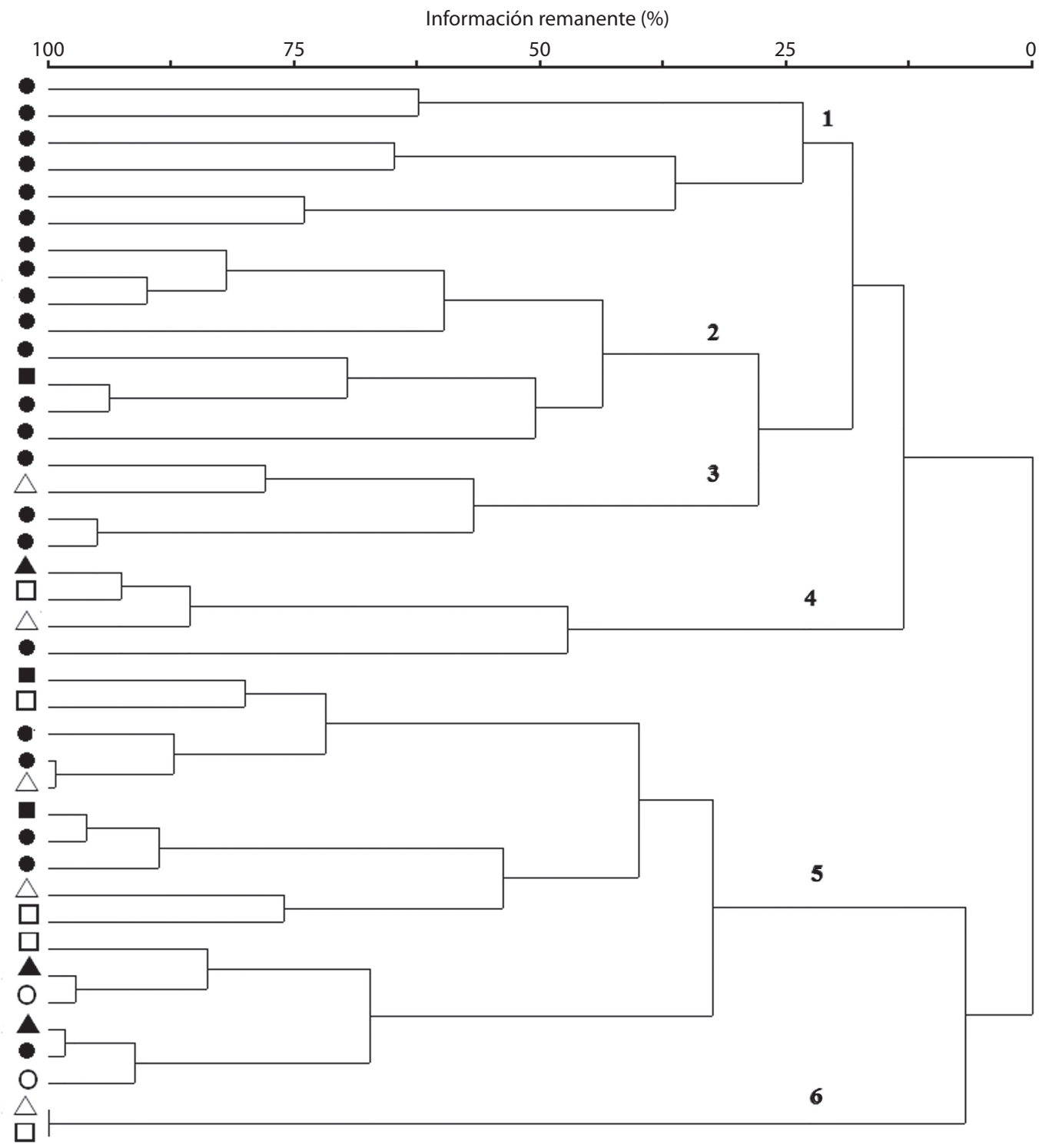

Fig. 1. Agrupamiento de las unidades de muestreo con base en los valores de cobertura de las especies. $\bullet$ P. cembroides, ○ P. johannis, - P. cembroides-P. johannis, $\square$ P. cembroides-P. johannis-Quercus potosina, $\boldsymbol{\text { A }}$. cembroides-Q. potosina, $\triangle P$. johannis-Q. potosina

Fig. 1. Grouping of sampling units based on cover values of species. P. cembroides, ○ P. johannis, $\square$. cembroides $-P$. johannis, $\square$ P. cembroides-P. johannis-Quercus potosina, $\triangle$ P. cembroides-Q. potosina, $\triangle$ P. johannis-Q. potosina.

fimbriatum. El quinto grupo fue el más heterogéneo, e incluyó rodales mixtos en su mayor parte que asociaron a las dos especies de piñonero con el encinar, allí, las especies características fueron Piptochaetium brevicalix, $P$. fimbriatum, Perymenium mendezii, D. grahamii, L. phleoides, Stevia lucida y E. flavescens. El último grupo fue también mixto, pero cabe mencionar la presencia de $P$. johannis y $Q$. potosina en ambas asociaciones vegetales; 
estos rodales son los que presentaron menor número de especies entre las que sobresalieron Garrya ovata, T. micrantha y P. mendezii.

Procedimiento de permutación de respuesta múltiple: En el cuadro 1 quedaron representados los resultados del procedimiento de permutación de respuesta múltiple. Lo que destacó es que entre los grupos (G) 1 a 3, que correspondieron a sitios preferentemente dominados por $P$. cembroides no existieron diferencias evidentes en la composición florística a juzgar por el valor de T, por lo cual aceptamos la hipótesis de no diferencias florísticas entre ellos; pero, cabe hacer notar que los valores más negativos de $\mathrm{T}$ se presentaron entre el G2 y G3 dominados por $P$. cembroides respecto a G5, bosques mixtos pero con sitios de $P$. johannis y encinares; claramente pudimos rechazar la hipótesis nula, y considerar que entre estos sitios, las diferencias estuvieron determinadas por la presencia y abundancia diferencial de las especies.

Ordenación: El análisis de escalamiento multidimensional mostró que el estrés final para la solución tridimensional de la ordenación fue de 16.8, mientras que la proporción de la varianza representada por los tres ejes de ordenación fue de $65.9 \%$. La prueba de Monte Carlo produjo un valor de $\mathrm{p}<0.0040$, para la solución tridimensional de la ordenación.
El diagrama de ordenación entre las 40 unidades de muestreo y los factores ambientales (Fig. 2), mostró que el primer eje de ordenación correlacionó positivamente con la altitud (0.729) y negativamente con la temperatura media anual (-0.417) y la orientación (-0.406); los ejes 2 y 3 mostraron bajos valores de correlación con las variables analizadas. En general, puede mencionarse la tendencia de los piñonares de $P$. cembroides a localizarse en sitios de menor altitud, con temperaturas medias anuales relativamente más altas propias del clima seco (BS) y en laderas con orientación principalmente al sur y suroeste del área de estudio; en los cuales, las especies características fueron: $J$. dioica, A. divaricata, T. micrantha, Bouteloua scorpioides, Microchloa kuntii, D. argentea, $B$. gracilis, Schizachirium sanguineum, Hechtia glomerata, Stevia salicifolia, Dasylirion acrotrichum y Agave lechuguilla. En contraste, los rodales de $P$. johannis, las masas mixtas de $P$. johannis- $P$. cembroides y de éstos en asociación con encinares, por lo regular, estuvieron ubicados en condiciones de mayor altitud, temperatura media anual ligeramente menor, aproximada al clima templado subhúmedo $(\mathrm{Cw})$, y con orientación al norte-noreste del área; en ellos, las especies más representativas fueron P. mendezii, P. fimbriatum, P. brevicalix, G. ovata, Commelina dianthifolia y S. lucida. Salvia axillaris, S. lucida, Heteropogon contortus y $C$. diversifolia.

CUADRO 1

Prueba de diferencias en la composición de especies de los grupos derivados del análisis de agrupamiento, con base en el procedimiento de permutación de respuesta múltiple. Bajo la diagonal se presentan los valores de $\mathrm{T}$ y sobre la diagonal el valor de $\mathrm{p}$

TABLE 1

Test of differences in the species composition of the groups derived from cluster analysis, based on the multiresponse permutation procedure. Below and upper diagonal are the values of $\mathrm{T}$ and $\mathrm{p}$ respectively

\begin{tabular}{ccccccc} 
& G1 & G2 & G3 & G4 & G5 & G6 \\
G1 & & 0.034 & 0.028 & 0.017 & 0.001 & 0.06 \\
G2 & -1.94 & & 0.003 & 0.01 & 0.000009 & 0.017 \\
G3 & -2.35 & -3.41 & & 0.0002 & 0.00000006 & 0.001 \\
G4 & -2.38 & -3.08 & -4.83 & & 0.00006 & 0.015 \\
G5 & -3.83 & -6.82 & -10.83 & -5.61 & & 0.0005 \\
G6 & -1.41 & -2.85 & -6.02 & -2.88 & -6.38 & \\
\hline
\end{tabular}




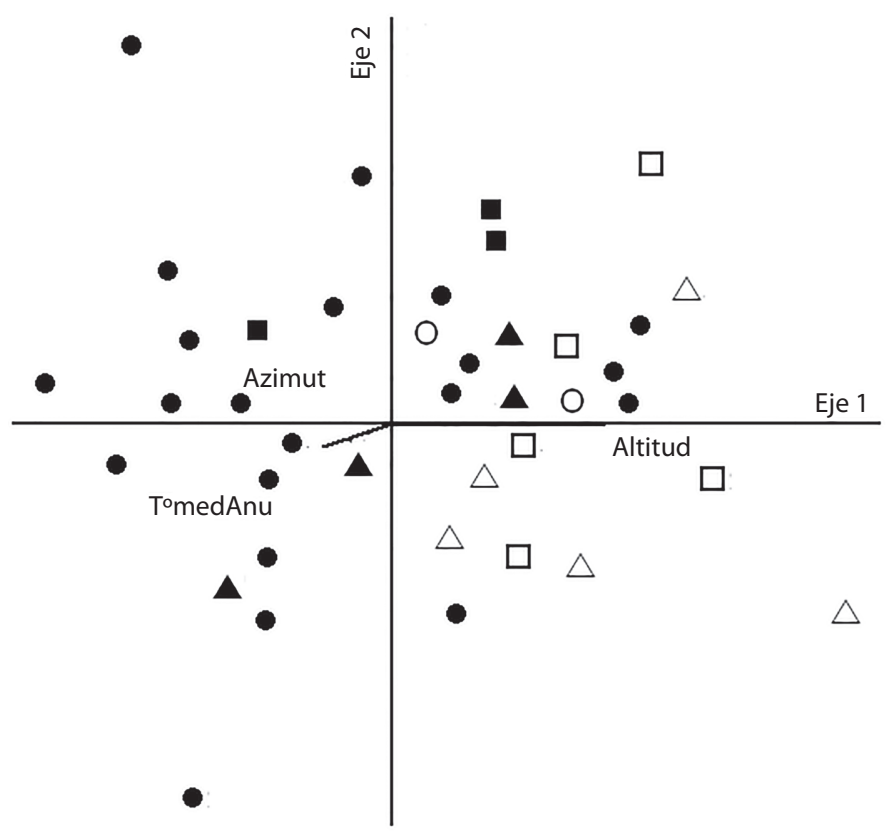

Fig. 2. Ordenación de las unidades de muestreo con base en los valores de cobertura de las especies. $\bullet P$. cembroides, $\bigcirc P$. johannis, $\square$. cembroides-P. johannis, $\square$ P. cembroides-P. johannis-Quercus potosina, $\triangle$ P. cembroides-Q. potosina, $\triangle P$. johannis-Q. potosina.

Fig. 2. Ordination of sampling units based on cover values of species. $\bullet$ P. cembroides, o P. johannis, $\square$. cembroides $-P$. johannis, $\square$ P. cembroides-P. johannis-Quercus potosina, $\triangle$ P. cembroides-Q. potosina, $\triangle$ P. johannis-Q. potosina.

La gráfica de ordenación mostró el efecto de la orientación de los sitios en la distribución de las especies; los sitios ubicados en los cuadrantes del lado izquierdo se relacionaron con las especies que preferentemente habitaron en las áreas de pendiente más suaves, menor altitud, laderas con orientación sur y oeste, ambientes secos con transición a mésicos, en bosques puros donde $P$. cembroides alcanzó las mayores densidades. Los sitios ubicados en el cuadrante superior derecho se asociaron con especies propias de una situación más mésica que xérica, generalmente con una exposición norte a este, las cuales estuvieron presentes bajo doseles de $P$. johannis como especie indicadora, aunque los bosques fuesen mixtos. El cuadrante inferior derecho mostró un conjunto de sitios con especies características de pendientes pronunciadas, con tendencia mésica y doseles mixtos de piñoneros, generalmente de $P$. johannis y encinos.

\section{DISCUSIÓN}

De acuerdo con McCune \& Grace (2002), la mayoría de los conjuntos de datos de comunidades ecológicas tendrían soluciones con valores de estrés entre 10-20, así que los resultados producto de este análisis de ordenación (estrés=16.8) fueron confiables al interpretar la relación entre las unidades de muestreo.

Las familias florísticas mejor representadas en los piñonares del Altiplano Potosino Zacatecano fueron Asteraceae, Poaceae, Lamiaceae, Fabaceae, Euphorbiaceae y Cyperaceae, también comunes en piñonares de Pinus pinceana Gordon \& Glend. (VillarrealQuintanilla, Mares-Arreola, Cornejo-Oviedo, \& Capó-Arteaga, 2009). Las 91 especies registradas en los transectos conformaron una lista amplia de especies para la zona, después de que Rebolledo (1982) consignó 52 especies y Ávila (1985) 73 especies. Hernández-Reyna \& 
García-Moya (1985), en contraste, registraron 125 especies. Para el bosque de P. johannis de Concepción del Oro, Zacatecas, Aldrete (1981) citó 17 especies, mientras que González (1998) consignó 52 especies. En la Sierra de Monte Grande-Charcas, San Luis Potosí, Reyes, González \& García (1996) registraron en piñonar de P. johannis, 78 especies, riqueza florística que superó a la observada en La Amapola, San Luis Potosí.

Fue posible agrupar conjuntos que muestran mayor o menor semejanza florística. De acuerdo con Tuomisto, Ruokolainen, \& Yli-Halla (2003) la baja semejanza florística entre sitios sería indicadora de condiciones ambientales diferentes. Los grupos 1-3 del dendrograma incluyeron a un conjunto de especies características de los bosques abiertos, de ecotonía, donde concurrieron los zacatales de las planicies con el límite arbolado de las faldas de las sierras. Varias de las especies allí encontradas como B. gracilis, B. scorpioides, $A$. divaricata y $D$. bicolor fueron comunes en áreas de bosques de piedemonte con tendencia xérica, es decir, fueron afines a climas secos (BS), donde destacaron elementos crasos bajo dosel de $P$. cembroides, principalmente hacia las exposiciones sur y suroeste, dada la convergencia con el matorral xerófilo.

En los otros grupos del dendrograma se ubicaron las especies de bosques densos que se presentan en sitios de mayor altitud, que van de piedemonte a escarpas, característicamente sobre terrenos gravosos, con temperaturas ligeramente más frías y quizá de mayor humedad, afines al clima templado subhúmedo $(\mathrm{Cw})$, donde se localizan $C$. dianthifolia, E. flavescens y diversas especies de Stevia. Dodonaea viscosa estuvo presente y fue una especie que Rzedowski (1978) calificó como indicadora de fuego. Los piedemontes de La Amapola fueron las áreas más alteradas por apacentamiento de caprinos, como también lo comentaron Huber-Sannwald, Maestre, Herrick \& Reynolds (2006) y Braasch (2012). Ahí aparecieron como especies indicadoras $A$. divaricata o $B$. scorpioides. Esta última especie ha sido una planta resistente al apacentamiento y a la sequía (Alcocer, Robberecht \& Thill, 1989). $P$. brevicalyx pudiese ser la herbácea fiel al bosque de pino-encino, algo más mésico.

$P$. cembroides fue una especie ubicua, mientras que $P$. johannis ocurrió como especie característica; a esta última se encuentra asociado $Q$. potosina, junto con plantas de estratos intermedios como Echeandia flavescens y $E$. mexicana, $C$. dianthifolia, Commelina tuberosa, Salvia ballotiflora, Salvia lycioides, S. lucida y $S$. viscida. Por su composición, las especies de los grupos 5 y 6 fueron características de condiciones mésicas, indicado por la presencia de $P$. johannis que se distribuyó preferentemente en las exposiciones norte y noreste, con alta cobertura arbórea, como lo indicó su asociación con $Q$. potosina, sujetas a quemas ocasionales. Barton (1993) ya se había referido a Pinus discolor D.K. Bailey \& Hawksw. (sinónimo de P. johannis) como una especie tolerante a la sombra y suelos cubiertos por una gruesa capa de hojarasca, aunque se ha visto intolerante al fuego por lo delgado de su corteza. Al igual que nuestros resultados, Aldrete \& Aguirre (1982) encontraron diferencias florísticas entre el bosque de $P$. cembroides y el de $P$. johannis de Concepción del Oro, Zacatecas.

Los resultados de la ordenación revelaron que el eje 1 correlacionó positivamente con la altitud y separó unidades de muestreo de $P$. cembroides, de menor altitud, de aquellas de $P$. johannis y de la asociación de ésta con $Q$. potosina, ubicadas a mayor altitud. Debido a la posición altitudinal, la topografía abrupta del terreno, pendientes superiores a $30^{\circ}$, y características del dosel de las especies concurrentes, se encontraron diferencias de sitio relevantes en variables hidrológicas, notándose a la vez, que las especies se posicionan a lo largo de otro gradiente, el cual correspondió con la humedad retenida por el suelo. De acuerdo con PérezSuárez, Arredondo-Moreno, Huber-Sannwald \& Serna-Pérez (2013), Q. potosina en La Amapola, S.L.P., con un dosel de hoja ancha, depositó el triple de hojarasca que $P$. cembroides $\mathrm{y}$, por esta diferencia, la cantidad de mantillo del suelo del encinar ha exhibido $15 \%$ más 
infiltración y $62 \%$ más capacidad de retención de agua que los sitios del piñonar, los que han mostrado solo $46 \%$ de retención de humedad; por lo mismo, el escurrimiento ha sido siete veces menor en los sitios de $Q$. potosina que en los de P. cembroides.

Al igual que en nuestro trabajo, EstradaCastillón, Jurado, Navar, Jímenez-Pérez \& Garza-Ocañas (2003) encontraron que gramíneas como Bouteloua fueron más frecuentes en terrenos de menor altitud, donde $B$. gracilis, $B$. hirsuta y $B$. scorpioides se asociaron preferentemente a los piñonares de $P$. cembroides; $B$. gracilis ha sido la gramínea prístina del pastizal del Desierto Chihuahuense, la unidad fisiográfica donde se ubica La Amapola S.L.P., que ha ocupado los sitios abiertos, de baja altitud y suelos algo húmedos (Yeaton \& Flores, 2009), sujetos a moderada intensidad de apacentamiento; otras gramíneas como $P$. fimbriatum y $M$. pubescens se han registrado asociadas a piñonares de $P$. johannis o $P$. johannis- $Q$. potosina, ubicados a mayor altitud.

Orientación, inclinación de la pendiente del sitio y humedad del suelo, son otros factores del medio que han influido significativamente en la distribución de la vegetación (Parker, 1991; Crowell \& Lane, 2001; Bataineh et al., 2007); en concordancia con lo anterior, la pendiente y la orientación tuvieron fuerte influencia en el contenido de humedad del suelo, ya que las pendientes orientadas al sur presentan mayor estrés calórico y baja humedad disponible que sus contrapartes orientadas al norte, caso que ya fue demostrado por Hubber-Sannwald et al. (2012) para La Amapola. Lo anterior probablemente explicaría el hecho de que en el área forestal estudiada hayamos registrado flora indicadora de sitios más secos como son Opuntia robusta y Agave, principalmente en laderas de orientación sur. Aun cuando en nuestro trabajo la radiación solar y el índice de calor aparentemente no mostraron una relación estrecha sobre la distribución y abundancia de las especies, estas variables han ejercido influencia en las condiciones ambientales, puesto que es sabido que las laderas con exposición sur han sido receptoras de mayor radiación solar y han almacenado menor humedad que las orientadas al norte (Nobel, 1982; Auslander, Nevo \& Inbar, 2003). Encontramos que $D$. argentea fue una especie heliófita común de sitios abiertos, similar a lo indicado por Passini (1982) y Bataineh et al. (2007). Arctostaphylos, Garrya, Arbutus, Rhus, Brickellia, Salvia y C. dianthifolia, se asociaron al bosque de transición mésico-xérica. Passini (1982) caracterizó a $C$. dianthifolia como especie indicadora de sitios con temperatura media anual de 12 a $16^{\circ} \mathrm{C}$, y en otro caso, Microchloa kunthii y Helianthemum patens, como propias de sitios con $600-700 \mathrm{~mm}$ de precipitación, es decir, de medios secos con tendencia a mésico.

Es probable que otros factores edáficos pudiesen estar influyendo en las relaciones florísticas de los piñonares, como la textura franco-limosa y la materia orgánica 2.2-8.3\% en Mesa La Carbonera (hacia el norte del área), donde domina $P$. cembroides, o la textura arenosa y $1.27 \%$ de materia orgánica del pastizal natural, situado en los piedemonte y planicies, más representativo hacia Aldana, al noreste del área. La diferencia entre ambas micro-regiones ha reflejado, como lo habíamos supuesto, que hacia las partes altas del bosque ha habido mejor condición ecosistémica que en las partes bajas; como lo han demostrado Huber-Sannwald et al. (2012), quienes cuantificaron para las zonas de mayor altura, características como mayor cobertura, infiltración de agua y mejor calidad del suelo en términos de materia orgánica, carbono, nitrógeno y mineralización del nitrógeno, que en las partes de menor altitud.

La falta de concordancia en la asociación de especies acompañantes de los piñoneros, inicialmente planteada por Romero-Manzanares et al. (1989) fue superada mediante el uso de técnicas multivariables, las cuales dieron mayor resolución para confirmar las diferencias en la composición florística de los diferentes bosques, y asegurar que, aunque la cubierta arbórea parezca la misma por la fisonomía de los piñoneros, existen agrupamientos preferenciales entre las especies, los cuales definen la composición del bosque. Las diferencias 
florísticas entre los bosques de $P$. cembroides y $P$. johannis pueden ser importantes para generar opciones de aprovechamiento o prácticas adecuadas de rehabilitación y conservación de áreas, ya que las especies indicadoras han sido clave para predeterminar diferencias de sitio.

En nuestro estudio, varias unidades de muestreo de P. cembroides y P. johannis se asociaron en el agrupamiento y la ordenación, lo cual ha sugerido una alta semejanza florística, debida en parte, a la simpatría de las especies de piñonero en términos fitogeográficos, y también, a las ecotonías donde convergen a escala local ambas especies y sus acompañantes asociadas; consideramos importante insistir en que, entre piñonares monoespecíficos, hubo claro efecto diferencial de los factores ambientales. Aparte de la influencia de los factores topográficos, de temperatura e incidencia solar ya discutidos, las diferencias en la flora de los piñonares también han dependido del tipo de sustrato, puesto que, $P$. johannis ha estado restringido a sustratos sedimentarios, mientras que $P$. cembroides ha sido preferente, más no ha sido de distribución obligada, al sustrato ígneo (Romero-Manzanares, García-Moya \& Passini, 1996).

Las técnicas multivariables mostraron que sí hubo diferencia entre la composición florística de los bosques de $P$. cembroides y la de $P$. johannis, incluso con los bosques mixtos o las transiciones hacia los encinares arbustivos; la localización de estos bosques monoespecíficos y mixtos de piñonero junto con las especies asociadas, algunas indicadoras de variables ambientales, serían una fuente de información importante para generar el pre-diagnóstico de la situación actual de las comunidades, para propósitos diversos, como los de estimación de la condición y manejo acordes con el balance natural ecosistémico.

\section{AGRADECIMIENTOS}

Los autores manifestamos nuestro agradecimiento a José García, por su ayuda en la determinación del material botánico. Juan Felipe Martínez y José Luis Flores quienes apoyaron en distintas actividades de campo. Nuestro reconocimiento a los revisores anónimos que con sus observaciones mejoraron sustancialmente este manuscrito.

\section{RESUMEN}

El conocimiento de los patrones de distribución de especies en las comunidades vegetales y su relación con factores del medio es un objetivo fundamental en ecología. Las comunidades de Pinus cembroides y P. johannis coexisten en diversas regiones de México, pero P. cembroides tiende a distribuirse en laderas de exposiciones sur y suroeste en sitios deteriorados, a diferencia de P. johannis localizados en áreas mésicas en laderas con exposición norte y noreste. El objetivo de este trabajo fue analizar la influencia de algunos factores físicos en la semejanza florística de los piñonares de $P$. cembroides y $P$. johannis. El área de estudio se ubica en la Sierra San Miguelito, San Luis Potosí, México. Se seleccionaron 40 unidades de muestreo, distribuidas en una superficie de $50 \mathrm{~km}^{2}$; en cada una se trazaron dos líneas de $20 \mathrm{~m}$ perpendiculares entre sí, donde se registró la cobertura de los individuos interceptados. Se elaboraron dos tipos de matrices de datos: la primera con los valores de cobertura de 91 especies y la segunda, con siete variables topográficas, climáticas y de incidencia solar. Se aplicaron análisis de agrupamiento y de ordenación para explorar la influencia de variables ambientales en la diferenciación florística de los piñonares. El agrupamiento diferenció seis grupos; los tres primeros caracterizados por bosques de $P$. cembroides. La ordenación mostró que la varianza representada por los tres primeros ejes fue de $65.9 \%$. El eje uno se correlacionó positivamente con la altitud y negativamente con la temperatura media anual y la orientación; los ejes dos y tres mostraron bajos valores de correlación con las variables probadas. Se observó que los piñonares de $P$. cembroides y flora acompañante, tienden a localizarse en sitios de menor altitud, con mayor temperatura media anual y en laderas con orientación al sur y suroeste. En contraste, los sitios de $P$. johannis, los de $P$. johannis- $P$. cembroides y de éstos con Quercus potosina, por lo regular están ubicados en condiciones de mayor altitud, temperatura media anual baja y con orientación al norte-noreste. La localización de estos bosques monoespecíficos y mixtos junto con las especies asociadas, indicadoras de variables ambientales, genera el pre-diagnóstico de la situación de las comunidades y su condición, para que en el futuro se planifiquen acciones acordes con el balance natural ecosistémico.

Palabras clave: piñonares, flora, agrupamiento, ordenación, especies indicadoras. 


\section{REFERENCIAS}

Alcocer, R., Robberecht, R., \& Thill, L. F. (1989). The response of Bouteloua scorpioides to water stress at two phonological stages. Botanical Gazette, 150(4), 454-461.

Aldrete, E. (1981). Estudio ecológico de los agostaderos del noreste del estado de Zacatecas. (Tesis profesional). Universidad Autonóma Chapingo, Chapingo, México.

Aldrete, E. \& Aguirre, J. R. (1982). Diferenciación de sitios y condición de agostaderos del noreste del estado de Zacatecas. Revista Chapingo, 35-36, 53-58.

Auslander, M., Nevo, E., \& Inbar, M. (2003). The effects of slope orientation on plant growth, developmental instability and susceptibility to herbivores. Journal of Arid Environments, 55, 405-416.

Ávila, A. (1985). Caracterización de los piñoneros Pinus cembroides Zucc. y P. discolor Bailey \& Hawks., de las serranias meridionales del estado de San Luis Potosí, México. (Tesis profesional). Universidad Michoacana de San Nicolás de Hidalgo, Morelia, México.

Barton, A. M. (1993). Factors controlling plant distributions: drought, competition, and fire in montane pines in Arizona. Ecological Monographs, 63, 367-397.-

Bataineh, M. M., Oswald, B. P., Bataineh, A. L., Farrish, K. W., Coble, D. W., \& Edminster, C. B. (2007). Plant communities associated with Pinus ponderosa forests in the sky islands of the Davis Mountains, Texas. Journal of the Torrey Botanical Society, 134(4), 468-478.

Beals, E. W. (1984). Bray Curtis ordination: an effective strategy for analysis of multivariate ecological data. Advances in Ecological Research, 14, 1-55.

Birks, H. J. B. (1987). Recent methodological developments in quantitative descriptive biogeography. Annales Zoologici Fennici, 24, 165-178.

Braasch, M. (2012). Modelación de la Producción Primaria Neta en un bosque semiárido con disturbio antropogénico en San Luis Potosí, México. (Tesis de Maestro en Ciencias). Instituto Potosino de Investigación Científica y Tecnológica, A. C. (IPICYT), San Luis Potosí, México.

Clarke, K. R. (1993). Non-parametric multivariate analysis of changes in community structure. Australian Journal of Ecology, 18(1), 117-143.

Comisión Nacional para el Conocimiento y Uso de la Biodiversidad - CONABIO. (1997). "Provincias biogeográficas de México”. Escala 1: 4000 000. México.

Crowell, M. D. \& Lane, S. N. (2001). Evaluating interactions between soil drainage and seedling performance in a restoration of Pinus sylvestris woodland,
Scotland. Global Ecology and Biogeography, 10, 147-160

Estrada-Castillón, E., Jurado, E., Navar, J. J., JímenezPérez, J., \& Garza-Ocañas, F. (2003). Plant associations of Cumbres de Majalca National Park, Chihuahua, México. Southwestern Naturalist, 48(2), 177-187.

FAO/UNESCO/ISRIC. (1998). Mapa de suelos dominantes, Escala 1: 4000 000, Primera aproximación. SEMARNAP/CP/INEGI. México, D. F.

González, J. (1998). Los bosques piñoneros de México, estudio del bosque de Pinus johannis M-F. Robert, en Concepción del Oro Zacatecas. (Tesis de Maestría). Universidad Autónoma Chapingo, México.

Hernández-Reyna, A. \& García-Moya, E. (1985). Análisis estructural de los piñonares del altiplano potosino. Agrociencia, 62, 7-20.

Huber-Sannwald, E., Maestre, F. T., Herrick, J. E., \& Reynolds, J. F. (2006). Ecohydrological feedbacks linkages associated with land degradation: a case study from Mexico. Hydrological Processes, 20, 3395-3411.

Huber-Sannwald, E., Ribero, M., Arredondo, J. T., Braasch, M., Martínez, R. M., García, J., \& Monsalvo, K. (2012). Navigating challenges and opportunities of land degradation and sustainable livelihood development in dryland social-ecologial systems: a case study from Mexico. Philosophical Transactions of The Royal Society of London Series B-Biological Sciences, 367, 3158-3177.

INEGI (Instituto Nacional de Estadística, Geografía e Informática). (2002). Síntesis de Información Geografía del estado San Luís Potosí, primera edición. México: Instituto Nacional de Estadística, Geografía e Informática.

Justus, J. \& Sarkar, S. (2002). The principle of complementarity in the design of reserve networks to conserve biodiversity: A preliminary history. Journal of Biosciences, 27, 421-435.

Korb, J. E., Daniels, M. L., Laughlin, D. C., \& Fule, P. Z. (2007). Understory communities of warmdry, mixed-conifer forests in southwestern Colorado. Southwestern Naturalist, 52(4), 493-503.

Kruskal, J. B. (1964). Nonmetric multidimensional scaling: a numerical method. Psychometrika, 29, 115-129.

Labarthe, G., Tristán, M., \& Aranda, J. (1982). Revisión estratigráfica del Cenozoico de la parte central del estado de San Luis Potosí. Folleto Técnico No. 85, Instituto de Geología y Metalurgia, Universidad Autónoma de San Luis Potosí, San Luis Potosí, México.

Lanner, R. M. (1981). The piñon pine. Natural and cultural history. Nevada, USA.: University of Nevada Press. 
Légaré, S., Bergeron, Y., Leduc, A., \& Paré, D. (2001). Comparison of the understory vegetation in boreal forest types of Southwest Quebec. Canadian Journal of Botany, 79, 1019-1027.

Lesica, P., McCunne, B., Cooper, S. V., \& Hong, W. S. (1991). Differences in lichen ad bryophyte communities between old growth and managed second-growth forests in the Swan Valley, Montana. Canadian Journal of Botany, 69, 1745-1755.

McCune, B. \& Grace, J. B. (2002). Analysis of ecological communities. Gleneden Beach, Oregon, USA.: MjM Software Design.

McCune, B. \& Mefford, M. J. (2011). PC-ORD. Multivariate analysis of ecological data, versión 6. Gleneden Beach, Oregon, USA.: MjM Software.

McLaughlin, S. P. (1994). Floristic plant geography: the classification of floristic areas and floristic elements. Progress in Physical Geography, 18, 185-208.

Mielke, P. W. (1991). The application of multivariate permutation methods based on distance functions in the earth sciences. Earth-Science Reviews, 31(1), 55-71.

Moora, M., Daniell, T., Kalle, H., Liira, J., Püssa, K., Roosaluste, E., Öpik, M., Wheatley, R., \& Zobel, M. (2007). Spatial pattern and species richness of boreonemoral forest understorey and its determinants-A comparison of differently managed forests. Forest Ecology and Management, 250, 64-70.

Nobel, P. S. (1982). Orientation, PAR interception, and nocturnal acidity increases for terminal cladodes of a widely cultivated cactus, Opuntia ficus-indica. American Journal of Botany, 69, 1462-1469.

Parker, K. C. (1991). Topography, substrate, and vegetation patterns in the northern Sonoran Desert. Journal of Biogeography, 18, 151-163.

Passini, M. F. (1982). Les forêts de Pinus cembroides au Mexique. Mission Archaeological e ethnologique Française au Mexique, Études Mesoaméricaines II-5. Paris: Editions Recherche sur les Civilisations.

Passini, M. F. (1994). Relèves floristiques des forêts de pins pignons au Mexique. Paris: Université Pierre et Marie Curie.

Peck, J. E. (2010). Multivariate analysis for community ecologists: Step by step using PC-ORD. Gleneden Beach, Oregon, USA: MjM Software Design.

Pérez-Suárez, M., Arredondo-Moreno, J. T., Huber-Sannwald, E., \& Serna-Pérez, A. (2013). Forest structure, species traits and rain characteristics influences on horizontal and vertical rainfall partitioning in a semiarid pine-oak forest from Central Mexico. Ecohydrology. doi: 10.1002/eco.1372

Rebolledo, A. (1982). Estudio preliminar sobre la ecología de los piñonares en el Altiplano Potosino-Zacatecano. (Tesis de Maestría). Colegio de Postgraduados, Chapingo, México.

Reyes, J. A., González, F., \& García, J. (1996). Flora vascular de la Sierra Monte Grande, municipio de Charcas, S.L.P., México. Boletín de la Sociedad Botánica de México, 58, 31-42.

Romero-Manzanares, A., García-Moya, E., Luna-Cavazos, M., \& De la Rosa-Vázquez, A. (1989). Especies indicadoras de sitios con potencial de producción de piñón en San Luis Potosí. Memorias del III Simposio Nacional sobre Pinos Piñoneros. Universidad Autónoma Agraria Antonio Narro, Saltillo, México.

Romero-Manzanares, A., García-Moya, E., \& Passini, M. F. (1996). Pinus cembroides s.l. y Pinus johannis del Altiplano Mexicano: una síntesis. Acta Botanica Gallica, 143, 681-693.

Romero-Manzanares, A., Aguirre-Rivera, R., \& GarcíaMoya, E. (1999). El papel del hombre en la evolución de los piñoneros Americanos. Agrociencia, 33, 473-482.

Rzedowski, J. (1978). La vegetación de México. México, D.F.: Limusa.

Sardinero, S. (2000). Classification and ordination of plant communities along an altitudinal gradient on the Presidential Range, New Hampshire, USA. Plant Ecology, 148, 81-103.

ter Braak, C. J. F. \& Prentice, I. C. (1988). A theory of gradient analysis. Advances in Ecological Research, 18, 271-317.

Tuomisto, H., Ruokolainen, K., \& Yli-Halla, M. (2003). Dispersal, environmental, and floristic variation of western Amazonian forests. Science, 299, 241-244.

Villarreal-Quintanilla, J. A., Mares-Arreola, O., CornejoOviedo, E., \& Capó-Arteaga, M. A. (2009). Estudio florístico de los piñonares de Pinus pinceana Gordon. Acta Botánica Mexicana, 89, 87-124.

Wishart, D. (1969). An algorithm for hierarchical classification. Biometrics, 25, 165-170.

Yeaton, R. I. \& Flores, J. L. (2009). Community structure of a Southern Chihuahuan Desert grassland under different grazing pressures. South African Journal of Botany, 75, 510-517. 


\section{APÉNDICE}

Lista de especies ordenado alfabéticamente, por estrato (E), con base en el perfil de alturas acotadas por Passini (1994).

E1, plantas de 1-5cm; E2, plantas de 5-25cm; E3, plantas de 25-50cm; E4, plantas de 50-100 cm; E5, plantas de 100$200 \mathrm{~cm}$; E6, plantas de $200-400 \mathrm{~cm}$; E7, plantas de $400-800 \mathrm{~cm}$

\section{APPENDIX}

Species list arranged alphabetically, by stratum (E), based on height profile mentioned by Passini (1994). E1, plants of $1-5 \mathrm{~cm}$; E2, plants of 5-25cm; E3, plants of $25-50 \mathrm{~cm}$; E4, plants of 50-100 cm; E5, plants of 100-200cm; E6, plants of 200$400 \mathrm{~cm}$; E7, plants of $400-800 \mathrm{~cm}$

\begin{tabular}{|c|c|c|c|c|c|c|c|}
\hline Especie & E1 & E2 & E3 & E4 & E5 & E6 & E7 \\
\hline Agave lechuguilla Torr. & & & & + & & & \\
\hline Agave parrasana Berger & & & & & + & & \\
\hline Agave salmiana Otto ex Salm-Dick & & & & & + & & \\
\hline Agave schidigera Lem. & & & + & & & & \\
\hline Ageratum corymbosum Zucc. & & & & + & & & \\
\hline Allium glandulosum Link \& Otto & & & + & & & & \\
\hline Aristida divaricata Humb. \& Bonpl. ex Willd. & & + & & & & & \\
\hline Aristida havardii Vasey & & & & + & & & \\
\hline Aristida schiedeana Trin. \& Rupr. & & & & + & & & \\
\hline Astrolepis sinuata (Lag. ex Sw.) D.M. Benham \& Windham & & & + & & & & \\
\hline Baccharis occidentalis S. F. Blake & & & & + & & & \\
\hline Bidens odorata Cav. & & & & + & & & \\
\hline Bouteloua curtipendula (Michx.) G. S. Torr. & & & & + & & & \\
\hline Bouteloua gracilis (Kunth) Lag. ex Griffiths & & & + & & & & \\
\hline Bouteloua hirsuta Lag. & & & + & & & & \\
\hline Bouteloua scorpioides Lag. & & & + & & & & \\
\hline Bouvardia ternifolia (Cav.) Schltdl. & & + & & & & & \\
\hline Brickellia veronicifolia (Kunth.) A. Gray & & & & + & & & \\
\hline Bromus anomalus Rupr. ex Fourn. & & & & + & & & \\
\hline Cardionema ramosissimmum (Weinm.) A. Nelson \& J.F. Macbr. & + & & & & & & \\
\hline Cercocarpus montanus Raf. & & & & & + & & \\
\hline Chaetopappa ericoides (Torr.) G. L. Nesom. & & & & + & & & \\
\hline Cerdia virescens Moç. \& Sessé ex DC. & + & & & & & & \\
\hline Commelina dianthifolia Delile & & & + & & & & \\
\hline Commelina tuberosa $\mathrm{L}$. & & + & & & & & \\
\hline Crusea diversifolia (Kunth) W. R. Anderson & & & & + & & & \\
\hline Cyperus seslerioides Kunth & & + & & & & & \\
\hline Cyperus spectabilis Link & & + & & & & & \\
\hline Dahlia coccinea Cav. & & & & + & & & \\
\hline Dalea bicolor Humb \& Bonpl. ex Willd. & & & & + & & & \\
\hline Dalea lutea (Cav.) Willd. & & + & & & & & \\
\hline Dasylirion acrotrichum (Schiede) Zucc. & & & & & + & & \\
\hline Desmodium grahamii A. Gray & & + & & & & & \\
\hline Dichondra argentea Humb. \& Bonpl. ex Willd. & + & & & & & & \\
\hline Dodonaea viscosa (L.) Jacq. & & & & & + & & \\
\hline Drymaria arenarioides Humb. \& Bonpl. ex Schult. & & + & & & & & \\
\hline Echeandia flavescens (Schult. \& Schult. f. Cruden) & & & + & & & & \\
\hline Echeandia mexicana Cruden & & & + & & & & \\
\hline Echinocereus pectinatus (Scheidw.) Engelm. & & & & + & & & \\
\hline Eragrostis intermedia Hitchc. & & & + & & & & \\
\hline Euphorbia anychioides Boiss. & & + & & & & & \\
\hline Euphorbia macropus (Klotzsch \& Garcke) Boiss. & & + & & & & & \\
\hline Evolvulus alsinioides L. & & + & & & & & \\
\hline
\end{tabular}


APÉNDICE / APPENDIX

\begin{tabular}{|c|c|c|c|c|c|c|c|}
\hline Especie & E1 & E2 & E3 & E4 & E5 & E6 & E7 \\
\hline Garrya ovata Benth. & & & & & + & & \\
\hline Gibasis linearis (Benth.) Rohw. & & & + & & & & \\
\hline Gibasis pulchella (Kunth) Raf. & & & + & & & & \\
\hline Hechtia glomerata Zucc. & & & & + & & & \\
\hline Helianthemum glomeratum (Lag.) Lag. & & + & & & & & \\
\hline Helianthemum patens Hemsl. & & + & & & & & \\
\hline Heteropogon contortus (L.) P. Beauv. ex Roem. \& Schult. & & & & + & & & \\
\hline Ipomoea purpurea (L.) Roth & & & + & & & & \\
\hline Ipomoea stans Cav. & & & + & & & & \\
\hline Jatropha dioica Cerv. & & + & & & & & \\
\hline Loeselia caerulea (Cav.) G. Don & & & + & & & & \\
\hline Loeselia mexicana (Lam.) Brand & & & + & & & & \\
\hline Lycurus phleoides Kunth & & & + & & & & \\
\hline Mammillaria sp. & & + & & & & & \\
\hline Microchloa kunthii Desv. & & & + & & & & \\
\hline Mimosa minutifolia B. L. Rob. \& Greenm. & & & + & & & & \\
\hline Muhlenbergia dubia E. Fourn. & & + & & & & & \\
\hline Muhlenbergia pubescens (Kunth) Hitchc. & & & & + & & & \\
\hline Muhlenbergia quadridentata (Kunth) Trin. & & & & + & & & \\
\hline Muhlenbergia rigida (Kunth) Kunth & & & + & & & & \\
\hline Nissolia wislizenii (A. Gray) A. Gray & & + & & & & & \\
\hline Opuntia robusta J.C. Wendl. & & & & + & & & \\
\hline Painteria leptophylla (DC.) Britton \& Rose & & & + & & & & \\
\hline Perymenium mendezii DC. & & & & + & & & \\
\hline Physaria argyraea (A. Gray) O'Kane \& Al-Shehbaz & & + & & & & & \\
\hline Pinus cembroides Zucc. & & & & & & & + \\
\hline Pinus johannis M.F. Robert & & & & & & + & + \\
\hline Piptochaetium brevicalyx (E. Fourn.) Ricker & & + & & & & & \\
\hline Piptochaetium fimbriatum (Kunth) Hitchc. & & & + & & & & \\
\hline Piqueria trinervia Cav. & & & + & & & & \\
\hline Plantago nivea Kunth & & + & & & & & \\
\hline Polygala compacta Rose & & + & & & & & \\
\hline Quercus potosina Trel. & & & & & & + & \\
\hline Salvia axillaris Moc. \& Sessé & & & + & & & & \\
\hline Salvia ballotiflora Benth. & & & & + & & & \\
\hline Salvia lycioides A. Gray & & & & + & & & \\
\hline Salvia microphylla Kunth & & & & + & & & \\
\hline Salvia regla Cav. & & & & & + & & \\
\hline Schizachyrium sanguineum (Retz.) Alston & & & & + & & & \\
\hline Solanum stoloniferum Schltdl. & & & + & & & & \\
\hline Sporobolus pyramidatus (Lam.) Hitchc. & & & + & & & & \\
\hline Stevia lucida Lag. & & & & + & & & \\
\hline Stevia salicifolia Cav. & & & & + & & & \\
\hline Stevia tomentosa Kunth & & & & + & & & \\
\hline Stevia viscida Kunth & & & & + & & & \\
\hline Steviopsis thyrsiflora (A. Gray) B. L. Turner & & & & + & & & \\
\hline Tagetes micrantha Cav. & & + & & & & & \\
\hline Trachypogon spicatus (L.f.) Kuntze & & + & & & & & \\
\hline Vernonia schaffneri A. Gray & & & + & & & & \\
\hline Zornia thymifolia Kunth & & + & & & & & \\
\hline
\end{tabular}

